\title{
Percepción de las habilidades de convivencia escolar en la Enseñanza Universitaria: análisis comparativo de la validez de constructo entre España y Brasil
}

\author{
Juan A. Valdivieso ${ }^{1}$, Miguel Ángel Carbonero ${ }^{1}$, Luis Jorge Martín-Antón ${ }^{1}$ \\ y Alvaci Freitas ${ }^{2}$ \\ ${ }^{1}$ Universidad de Valladolid (España); ${ }^{2}$ Universidad Tiradentes (Brasil)
}

\begin{abstract}
En este artículo se hace una reflexión teórica y un análisis descriptivo de los perfiles psicoinstruccionales de los estilos de enseñanza del profesorado de Educación Superior, en el ámbito de las habilidades sociales y competencias directamente relacionadas con la promoción de la Convivencia Escolar. Se hace un análisis comparativo de la autopercepción de una serie de variables docentes vinculadas con las Habilidades Sociales con una muestra de profesores de universidades españolas y brasileñas. A partir de la construcción y validación una escala tipo Likert de la competencia docente autopercibida en la Enseñanza Universitaria (CEVES) se fundamenta un modelo conceptual de variables profesionales de la competencia docente de este nivel de enseñanza, basado en planteamientos integradores de la Psicología de la Instrucción y de la Psicología Positiva. Mediante el juicio de expertos y la aplicación piloto a una muestra de $N=205$ profesores de Educación Superior del ámbito de Humanidades y de las Ciencias Sociales, se han analizado de forma comparativa entre contextos universitarios de España y Brasil las autopercepciones en cada una de las variables y dimensiones configuradoras de la acción docente vertebradoras de la competencia social.
\end{abstract}

Palabras clave: Estilos de enseñanza, Educación superior, Escala evaluación docente, habilidades sociales, convivencia.

Perception of school life skills in university education: comparative analysis of the construct validity between Spain and Brazil. This paper makes a theoretical and a descriptive analysis of the psycho-instructional profiles of the teaching styles of Higher Education teachers about social skills and competences directly related to the promotion of school co-existence. The main objective is establishing the comparative analysis of university teaching self-perceived of a sample of teachers of Spanish and Brazilian universities. From the construction and validation of a Likert scale of self-perceived teaching competence in university teaching (CEVES) we develop a conceptual model of professional variables of teaching competence in the Higher Education level, based on integrative approaches of Instructional and Positive Psychology. Through an expert judgment process and sampling of $N=205$ Higher Education professors in the field of Humanities and Social Sciences, we analyzed the self-perceptions results comparatively between spanish and brazilian universities in each of the variables.

Key words: Eaching styles, Higher education, Teacher evaluation scale, social skills, coexistence.

Correspondencia: Miguel Ángel Carbonero. Departamento de Psicología. Facultad de Educación y Trabajo Social. Universidad de Valladolid. Campus Miguel Delibes, Paseo de Belén, 1. C.P. 47011. Valladolid (España). E-mail: carboner@ psi.uva.es 
La universidad es una institución que tiene como presupuesto básico la formación del profesorado, sea cual fuera el nivel educativo, y no puede mantener su estilo de enseñanza del pasado. Aunque, necesita del pasado, para no olvidar las transformaciones que exigen cambios en el actual sistema educativo del siglo XXI.

La Enseñanza Superior para la formación de profesionales en distintas áreas, en este siglo, exige una actuación del profesor (docente) que sepa usar metodologías en el proceso de enseñanza más atractivas, dinámicas y eficaces, donde el protagonista sea el alumno, profesor con estilos de enseñanza no sólo de contenidos, sino que prepare al alumno para los desafíos de la vida. En este contexto, Hervás (2005) comenta que los estilos de enseñanza es un contenido que interesa a quienes investigan nuevas propuestas en la educación, que respondan a las demandas de la sociedad moderna, o de la sociedad del conocimiento y que atienda a la pluralidad y a la enseñanza individualizada.

Los actuales desafíos y demandas que afectan a los centros educativos y al profesorado en los sistemas educativos surgen de las nuevas expectativas y necesidades de los entornos formativos (Normand y Derouet, 2011; Pesquero y otros, 2008; Romuald y Derouet, 2011), aspectos que deben ser tenidos en cuenta a la hora de replantear metodologías y sistemas formativos.

Por ello, resulta conveniente desarrollar evaluaciones continuas de las autopercepciones docentes (Guillén, 1992), tanto de su propia intervención como de la enseñanza en general. A este respecto, encontramos algunos estudios que se limitan a poner de relieve aspectos diferentes de la labor docente (Braskamp, Caulley y Costin, 1979; Marshall, Overall y Kesler, 1979; Ovejero y otros, 1982) y la mayoría de investigaciones sobre la evaluación del profesorado no contemplan los aspectos contextuales que puedan estar modulando las percepciones (Guillén, 1992) y, en consecuencia, las valoraciones que el profesorado realiza de sus actividades docentes, lo que nos parece importante de ser analizado si queremos conocer la realidad del acto instruccional.

Para evaluar los diferentes componentes de naturaleza subyacente, como pueden ser las actitudes, atribuciones y autopercepciones manifestadas por el docente, se usan distintos procedimientos siendo de los más utilizados las técnicas de autoinforme en formato de cuestionario, que evalúan los componentes motivacionales y los procesos de pensamiento que no son observables (Bainbridge, 1999; Maintier y Alaphilipee, 2006; Yu y Ding, 2009). Se emplea esta metodología de investigación por la facilidad de su diseño y la rápida administración e interpretación de los resultados (Gana y Brechenmacher, 2001; Torrano y González, 2004; Winne y Perry, 2000).

En este artículo se presenta una propuesta conceptual y metodológica de asesoramiento psicológico e instruccional integradora de la competencia docente del profesorado universitario en el ámbito de la gestión y desarrollo de las Habilidades Sociales para la promoción de la Convivencia Escolar, validada para la definición 
operativa de un perfil de estilo de enseñanza eficaz en la Enseñanza Superior, conformando una propuesta experimental de variables y competencias psicoinstruccionales organizadoras del proceso de enseñanza-aprendizaje en el contexto del Espacio Europeo de Educación Superior (EEES), de tal forma que se pueda vertebrar un modelo o perfil de enseñanza ajustado a las valoraciones propias del docente universitario en activo.

\section{MÉTODO}

\section{Participantes}

La muestra consistió en $N=205$ profesores de Educación Superior del ámbito de Humanidades y de las Ciencias Sociales. Los criterios de inclusión de dicho profesorado fueron: $1^{\circ}$ ) Tener experiencia en la enseñanza universitaria de, al menos, 3 cursos escolares. $2^{\circ}$ ) Estar vinculado a un centro de enseñanza universitaria como docente. $3^{\circ}$ ) Contestar correctamente a los ítems de los dos cuestionarios (CEVES y CEMEDEPU).

En España, la representación del profesorado fue de $N=102$, de varias Comunidades Autónomas. Sin embargo, como en la muestra total se obtenía un alto grado de varianza de las distintas áreas, se procedió a homogeneizar al máximo la representatividad de los mismos, seleccionando únicamente a los docentes del área de humanidades y que ejercían funciones de docencia.

En Brasil, la representación del profesorado fue de $N=103$, procedentes de tres Estados de la región Nordeste: los Estados de Pernambuco, Alagoas y Sergipe. Al igual que se hizo con la muestra del profesorado de España, se pasó a homogeneizar al máximo la representatividad del mismo seleccionando únicamente al profesorado del área de humanidades y que ejercía funciones de docencia.

\section{Instrumentos}

Se ha configurado un instrumento valorativo tipo Likert centrado en el análisis de las autopercepciones de las habilidades y competencias de enseñanza por parte del docente de Educación Superior que favorecen las situaciones de convivencia en el ámbito universitario y que ha sido denominado CEVES (Cuestionario de Evaluación de las Variables de Enseñanza Superior).

Cada una de las variables del modelo conceptual propuesto se ha operativizado en un nivel de concreción mayor, con el fin de aclarar los fenómenos atribucionales que se están investigando y así poder establecer la comunicación científica en forma no ambigua (McGuigan, 1996). En definitiva, se han integrado en cada una de las variables un conjunto de subvariables en un orden de operacionalización más concreto, a modo de indicadores de evaluación organizadores del constructo 
competencial del perfil de evaluación psicoinstruccional que se pretende instaurar. Así, la variable Habilidades Sociales englobaría las habilidades de vinculación afectiva, asertividad, empatía, liderazgo, convivencia y relaciones con el alumnado.

Figura 1. Representación de la operativización de las Habilidades Sociales docentes CEVES (2012)

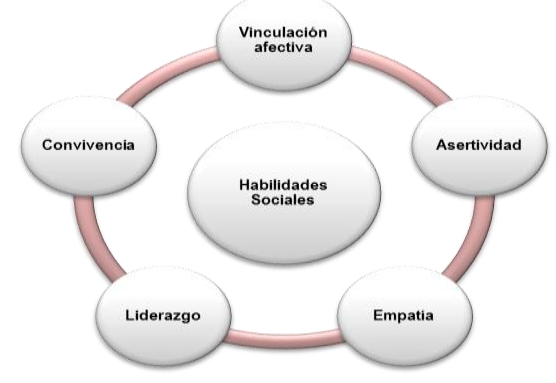

Otro instrumento empleado de forma complementaria fue el Cuestionario publicado por Gargallo-López y otros (2012): CEMEDEPU, con el fin de incrementar el constructo teórico de Estilo de Enseñanza universitario. Este Cuestionario se encuentra organizado en torno a tres variables docentes (procesos de enseñanza, procesos de aprendizaje y habilidades docentes del profesor eficaz) estableciendo tres escalas de medida, que cuentan con un índice de fiabilidad $\alpha$ Cronbach $=.879$ para la primera .382 para la segunda y .839 para la tercera.

\section{Diseño}

La metodología empleada ha seguido los descriptores propios de un estudio cuantitativo-comparativo, a partir de un enfoque empírico-analítico y con un diseño no experimental, psicométrico, de corte transversal con la utilización de un muestreo aleatorio-accidental (Kerlinger, 1985).

\section{Procedimiento}

Para la construcción y validación del instrumento de autoevaluación del docente universitario CEVES se procedió al diseño y análisis de validez de constructo y de contenido a partir de un Panel de Expertos compuesto por una muestra de profesorado de universidades de Brasil y otra muestra de profesorado de universidades de España.

La aplicación de ambos Cuestionarios (CEVES y CEMEDEPU) al Profesorado Universitario de los dos contextos (Brasil-España) se llevó a cabo on-line y de forma individual, habiendo sido traducidos a los idiomas oficiales de cada país.

Tras la recogida de datos del profesorado universitario que fue objeto de la aplicación, se procedió al análisis estadístico de tipo descriptivo-analítico mediante el 
paquete estadístico SPSS 19.0, concretándose en pruebas de análisis exploratorio y de comparación de medias, con la prueba no paramétrica U Mann-Whitney-Wilcoxon, al no cumplirse el supuesto de distribución normal de las puntuaciones.

\section{RESULTADOS}

Presentamos los análisis descriptivos de la principal variable Habilidades Sociales con sus respectivas dimensiones, que son: Vinculación afectiva, Asertividad, Empatía, Liderazgo, Convivencia y las Relaciones con el Alumnado, con las medias de España y Brasil, de acuerdo con el resultado del cuestionario CEVES. A continuación, en las tablas presentamos la validez de constructo y de contenido.

\section{Dimensión I. Vinculación Afectiva}

Presentamos los análisis descriptivos de Vinculación Afectiva, en las tablas 1 y 2, con sus respectivos ítems.

Tabla 1. Estadísticos descriptivos de la Dimensión I: Vinculación Afectiva

\begin{tabular}{ccccccccccc}
\hline \multirow{2}{*}{ N$^{\circ}$ Ítem } & \multicolumn{4}{c}{ Validez de Constructo (a) } & \multicolumn{6}{c}{ Validez de Contenido (b) } \\
\cline { 2 - 11 } & $N$ & Mín. & Máx. & $M$ & $S D$ & $N$ & Mín. & Máx. & $M$ & $S D$ \\
\hline 40 & 22 & 6 & 10 & 9.05 & 1.214 & 22 & 6 & 10 & 9.23 & 1.152 \\
\hline 41 & 22 & 5 & 10 & 9.18 & 1.181 & 22 & 5 & 10 & 9.23 & 1.193 \\
\hline 42 & 22 & 5 & 10 & 7.79 & 1.918 & 22 & 5 & 10 & 7.72 & 1.900 \\
\hline 43 & 22 & 6 & 10 & 8.45 & 1.371 & 22 & 5 & 10 & 8.45 & 1.438 \\
\hline 44 & 22 & 6 & 10 & 9.00 & 1.113 & 22 & 7 & 10 & 9.23 & .869 \\
\hline
\end{tabular}

Tabla 2. Estadísticos de la Dimensión I: Vinculación Afectiva (Comparación ES/BR)

\begin{tabular}{|c|c|c|c|c|c|c|c|c|c|c|c|}
\hline \multirow{2}{*}{$\begin{array}{l}\mathrm{N}^{\circ} \\
\text { Ítem }\end{array}$} & \multicolumn{6}{|c|}{ Validez de Constructo (a) } & \multicolumn{5}{|c|}{ Validez de Contenido (b) } \\
\hline & País & $N$ & $M$ & $S D$ & $U$ & Sig & $N$ & $M$ & $S D$ & $U$ & Sig \\
\hline \multirow{2}{*}{40} & España & 11 & 8.91 & 1.044 & \multirow{2}{*}{39.500} & \multirow{2}{*}{.171} & 11 & 9.00 & 1.095 & \multirow{2}{*}{35.500} & \multirow{2}{*}{.101} \\
\hline & Brasil & 11 & 9.18 & 1.401 & & & 11 & 9.45 & 1.214 & & \\
\hline \multirow{2}{*}{41} & España & 11 & 9.09 & 0.701 & \multirow{2}{*}{39.000} & \multirow{2}{*}{.171} & 11 & 9.18 & 0.751 & \multirow{2}{*}{43.500} & \multirow{2}{*}{.270} \\
\hline & Brasil & 11 & 9.27 & 1.555 & & & 11 & 9.27 & 1.555 & & \\
\hline \multirow{2}{*}{42} & España & 11 & 7.64 & 1.748 & \multirow{2}{*}{50.500} & \multirow{2}{*}{.519} & 11 & 7.63 & 1.612 & \multirow{2}{*}{52.000} & \multirow{2}{*}{.606} \\
\hline & Brasil & 11 & 8.00 & 2.145 & & & 11 & 7.81 & 2.228 & & \\
\hline \multirow{2}{*}{43} & España & 11 & 8.36 & 1.206 & \multirow{2}{*}{54.000} & \multirow{2}{*}{.699} & 11 & 8.64 & 0.924 & \multirow{2}{*}{60.000} & \multirow{2}{*}{1.000} \\
\hline & Brasil & 11 & 8.55 & 1.572 & & & 11 & 8.27 & 1.849 & & \\
\hline \multirow{2}{*}{44} & España & 11 & 8.73 & 1.272 & \multirow{2}{*}{45.500} & \multirow{2}{*}{.332} & 11 & 9.00 & 0.894 & \multirow{2}{*}{$41.500^{*}$} & \multirow{2}{*}{.217} \\
\hline & Brasil & 11 & 9.27 & 0.905 & & & 11 & 9.45 & 0.820 & & \\
\hline
\end{tabular}

\section{Dimensión II: Asertividad}

Presentamos los análisis descriptivos de Asertividad, en las tablas 3 y 4, con sus respectivos ítems. 
VALDIVIESO et al. Percepción de las habilidades de convivencia escolar...

Tabla 3. Estadísticos descriptivos de la Dimensión II: Asertividad

\begin{tabular}{|c|c|c|c|c|c|c|c|c|c|c|}
\hline \multirow{2}{*}{$\mathrm{N}^{\mathrm{o}}$ Ítem } & \multicolumn{5}{|c|}{ Validez de Constructo (a) } & \multicolumn{5}{|c|}{ Validez de Contenido (b) } \\
\hline & $N$ & Mín. & Máx. & $M$ & $S D$ & $N$ & Min. & Máx. & $M$ & $S D$ \\
\hline 45 & 22 & 5 & 10 & 8.68 & 1.359 & 22 & 5 & 10 & 8.68 & 1.555 \\
\hline 46 & 22 & 1 & 10 & 7.86 & 2.336 & 22 & 1 & 10 & 7.91 & 2.180 \\
\hline 47 & 22 & 6 & 10 & 9.00 & 1.195 & 22 & 6 & 10 & 8.91 & 1.377 \\
\hline 48 & 22 & 6 & 10 & 9.09 & 1.109 & 22 & 6 & 10 & 9.00 & 1.155 \\
\hline
\end{tabular}

Tabla 4. Estadísticos descriptivos de la Dimensión II: Asertividad (Comparación ES/BR)

\begin{tabular}{|c|c|c|c|c|c|c|c|c|c|c|c|}
\hline \multirow{2}{*}{$\begin{array}{l}\mathrm{N}^{\mathrm{o}} \\
\text { Ítem }\end{array}$} & \multicolumn{6}{|c|}{ Validez de Constructo (a) } & \multicolumn{5}{|c|}{ Validez de Contenido (b) } \\
\hline & País & $N$ & $M$ & $S D$ & $U$ & Sig & $N$ & $M$ & $S D$ & $U$ & Sig \\
\hline \multirow{2}{*}{45} & España & 11 & 8.27 & 1.489 & \multirow{2}{*}{40.000} & \multirow{2}{*}{.193} & 11 & 8.18 & 1.662 & \multirow{2}{*}{32.500} & \multirow{2}{*}{.065} \\
\hline & Brasil & 11 & 9.09 & 1.136 & & & 11 & 9.18 & 1.328 & & \\
\hline \multirow{2}{*}{46} & España & 11 & 7.18 & 1.834 & \multirow{2}{*}{$30.000 *$} & \multirow{2}{*}{.047} & 11 & 7.27 & 1.421 & \multirow{2}{*}{$24.500^{*}$} & \multirow{2}{*}{.016} \\
\hline & Brasil & 11 & 8.55 & 2.659 & & & 11 & 8.55 & 2.659 & & \\
\hline \multirow{2}{*}{47} & España & 11 & 8.18 & 1.079 & \multirow{2}{*}{$7.000 * * *$} & \multirow{2}{*}{.000} & 11 & 8.00 & 1.342 & \multirow{2}{*}{$7.000 * * *$} & \multirow{2}{*}{.000} \\
\hline & Brasil & 11 & 9.82 & 0.603 & & & 11 & 9.82 & 0.603 & & \\
\hline \multirow{2}{*}{48} & España & 11 & 8.64 & 1.027 & \multirow{2}{*}{$23.500^{*}$} & \multirow{2}{*}{.013} & 11 & 8.45 & 1.036 & \multirow{2}{*}{$18.000 * *$} & \multirow{2}{*}{.004} \\
\hline & Brasil & 11 & 9.55 & 1.036 & & & 11 & 9.55 & 1.036 & & \\
\hline
\end{tabular}

$* p<.05 * * p<.01 * * * p<.001$

\section{Dimensión III: Empatía}

Tabla 5. Estadísticos descriptivos de la Dimensión III: Empatía

\begin{tabular}{|c|c|c|c|c|c|c|c|c|c|c|}
\hline \multirow{2}{*}{$\mathrm{N}^{\mathrm{o}}$ Ítem } & \multicolumn{5}{|c|}{ Validez de Constructo (a) } & \multicolumn{5}{|c|}{ Validez de Contenido (b) } \\
\hline & $N$ & Mín. & Máx. & $M$ & $S D$ & $n$ & Mín. & Máx. & $M$ & $S D$ \\
\hline 49 & 22 & 4 & 10 & 8.95 & 1.676 & 22 & 4 & 10 & 8.77 & 1.798 \\
\hline 50 & 22 & 6 & 10 & 8.86 & 1.356 & 22 & 6 & 10 & 9.09 & 1.151 \\
\hline 51 & 22 & 4 & 10 & 8.45 & 1.711 & 22 & 5 & 10 & 8.50 & 1.683 \\
\hline 52 & 22 & 4 & 10 & 8.64 & 1.529 & 22 & 4 & 10 & 8.77 & 1.798 \\
\hline
\end{tabular}

Tabla 6. Estadísticos descriptivos de la Dimensión III: Empatía (Comparación ES/BR)

\begin{tabular}{|c|c|c|c|c|c|c|c|c|c|c|c|}
\hline \multirow{2}{*}{$\begin{array}{c}\mathrm{N}^{\mathrm{o}} \\
\text { Ítem }\end{array}$} & \multicolumn{6}{|c|}{ Validez de Constructo (a) } & \multicolumn{5}{|c|}{ Validez de Contenido (b) } \\
\hline & País & $N$ & $M$ & $S D$ & $U$ & Sig & $N$ & $M$ & $S D$ & $U$ & Sig \\
\hline \multirow{2}{*}{49} & España & 11 & 8.73 & 1.618 & \multirow{2}{*}{46.500} & \multirow{2}{*}{.365} & 11 & 8.36 & 1.804 & \multirow{2}{*}{41.500} & \multirow{2}{*}{.217} \\
\hline & Brasil & 11 & 9.18 & 1.779 & & & 11 & 9.18 & 1.779 & & \\
\hline \multirow{2}{*}{50} & España & 11 & 8.36 & 1.433 & \multirow{2}{*}{31.500} & \multirow{2}{*}{.056} & 11 & 8.64 & 1.286 & \multirow{2}{*}{33.000} & \multirow{2}{*}{.076} \\
\hline & Brasil & 11 & 9.36 & 1.120 & & & 11 & 9.55 & 0.820 & & \\
\hline \multirow{2}{*}{51} & España & 11 & 8.18 & 1.779 & \multirow{2}{*}{47.500} & \multirow{2}{*}{.401} & 11 & 8.18 & 1.722 & \multirow{2}{*}{42.000} & \multirow{2}{*}{.243} \\
\hline & Brasil & 11 & 8.73 & 1.679 & & & 11 & 8.82 & 1.662 & & \\
\hline \multirow{2}{*}{52} & España & 11 & 8.00 & 1.732 & \multirow{2}{*}{31.500} & \multirow{2}{*}{.056} & 11 & 8.36 & 1.690 & \multirow{2}{*}{32.000} & \multirow{2}{*}{.065} \\
\hline & Brasil & 11 & 9.27 & 1.009 & & & 11 & 9.45 & 0.934 & & \\
\hline
\end{tabular}

$* p<.05 * * p<.01 * * * p<.001$

Dimensión IV: Liderazgo

Tabla 7. Estadísticos descriptivos de la Dimensión IV: Liderazgo

\begin{tabular}{|c|c|c|c|c|c|c|c|c|c|c|}
\hline \multirow{2}{*}{$\mathrm{N}^{\circ}$ Ítem } & \multicolumn{5}{|c|}{ Validez de Constructo (a) } & \multicolumn{5}{|c|}{ Validez de Contenido (b) } \\
\hline & $N$ & Mín. & Máx. & $M$ & $S D$ & $N$ & Mín. & Máx. & $M$ & $S D$ \\
\hline 53 & 22 & 1 & 10 & 9.05 & 1.939 & 22 & 7 & 10 & 9.18 & 1.006 \\
\hline 54 & 22 & 1 & 10 & 9.32 & 1.912 & 22 & 7 & 10 & 9.45 & .858 \\
\hline 55 & 22 & 7 & 10 & 9.32 & 0.995 & 22 & 7 & 10 & 9.32 & .894 \\
\hline 56 & 22 & 8 & 10 & 9.45 & 0.739 & 22 & 7 & 10 & 9.36 & 1.002 \\
\hline
\end{tabular}


VALDIVIESO et al. Percepción de las habilidades de convivencia escolar...

Tabla 8. Estadísticos descriptivos de la Dimensión IV: Liderazgo (Comparación ES/BR)

\begin{tabular}{|c|c|c|c|c|c|c|c|c|c|c|c|}
\hline \multirow{2}{*}{$\begin{array}{l}\mathrm{N}^{\mathrm{o}} \\
\text { Îtem }\end{array}$} & \multicolumn{6}{|c|}{ Validez de Constructo (a) } & \multicolumn{5}{|c|}{ Validez de Contenido (b) } \\
\hline & País & $N$ & $M$ & $S D$ & $U$ & Sig & $N$ & $M$ & $S D$ & $U$ & Sig \\
\hline \multirow{2}{*}{53} & España & 11 & 8.36 & 2.541 & \multirow{2}{*}{$28.000 *$} & \multirow{2}{*}{.034} & 11 & 8.64 & 1.027 & \multirow{2}{*}{$21.500^{* * *}$} & \multirow{2}{*}{.008} \\
\hline & Brasil & 11 & 9.73 & 0.647 & & & 11 & 9.73 & 0.647 & & \\
\hline \multirow{2}{*}{54} & España & 11 & 8.73 & 2.611 & \multirow{2}{*}{32.500} & \multirow{2}{*}{.065} & 11 & 9.00 & 1.000 & \multirow{2}{*}{$26.000 *$} & \multirow{2}{*}{.023} \\
\hline & Brasil & 11 & 9.91 & 0.302 & & & 11 & 9.91 & 0.302 & & \\
\hline \multirow{2}{*}{55} & España & 11 & 8.91 & 1.136 & \multirow{2}{*}{32.500} & \multirow{2}{*}{.065} & 11 & 8.91 & 0.944 & \multirow{2}{*}{$28.000^{*}$} & \multirow{2}{*}{.034} \\
\hline & Brasil & 11 & 9.73 & 0.647 & & & 11 & 9.73 & 0.647 & & \\
\hline \multirow{2}{*}{56} & España & 11 & 9.27 & 0.786 & \multirow{2}{*}{44.000} & \multirow{2}{*}{.300} & 11 & 9.00 & 1.183 & \multirow{2}{*}{37.500} & \multirow{2}{*}{.133} \\
\hline & Brasil & 11 & 9.64 & 0.674 & & & 11 & 9.73 & 0.647 & & \\
\hline
\end{tabular}

Dimensión V: Convivencia

Tabla 9. Estadísticos descriptivos de la Dimensión V: Convivencia

\begin{tabular}{|c|c|c|c|c|c|c|c|c|c|c|}
\hline \multirow{2}{*}{$\mathrm{N}^{o}$ Ítem } & \multicolumn{5}{|c|}{ Validez de Constructo (a) } & \multicolumn{5}{|c|}{ Validez de Contenido (b) } \\
\hline & $N$ & Mín. & Máx. & $M$ & $S D$ & $n$ & Mín. & Máx. & $M$ & $S D$ \\
\hline 57 & 22 & 6 & 10 & 9.18 & 1.259 & 22 & 6 & 10 & 9.23 & 1.110 \\
\hline 58 & 22 & 8 & 10 & 9.68 & 568 & 22 & 8 & 10 & 9.64 & .581 \\
\hline 59 & 22 & 7 & 10 & 9.41 & .908 & 22 & 7 & 10 & 9.23 & .922 \\
\hline 60 & 22 & 4 & 10 & 8.82 & 1.563 & 22 & 4 & 10 & 8.41 & 2.085 \\
\hline 61 & 22 & 7 & 10 & 9.50 & .802 & 22 & 6 & 10 & 9.23 & 1.110 \\
\hline
\end{tabular}

Tabla 10. Estadísticos descriptivos de la Dimensión V: Convivencia (Comparación ES/BR)

\begin{tabular}{|c|c|c|c|c|c|c|c|c|c|c|c|}
\hline \multirow{2}{*}{$\begin{array}{l}\mathrm{N}^{\circ} \\
\text { Ítem }\end{array}$} & \multicolumn{6}{|c|}{ Validez de Constructo (a) } & \multicolumn{5}{|c|}{ Validez de Contenido (b) } \\
\hline & País & $N$ & $M$ & $S D$ & $U$ & Sig & $N$ & $M$ & $S D$ & $U$ & Sig \\
\hline \multirow{2}{*}{57} & España & 11 & 8.73 & 1.421 & \multirow{2}{*}{$28.500 *$} & \multirow{2}{*}{.034} & 11 & 8.73 & 1.104 & \multirow{2}{*}{$20.000^{* *}$} & \multirow{2}{*}{.007} \\
\hline & Brasil & 11 & 9.64 & 0.924 & & & 11 & 9.73 & 0.905 & & \\
\hline \multirow{2}{*}{58} & España & 11 & 9.45 & 0.688 & \multirow{2}{*}{38.000} & \multirow{2}{*}{.151} & 11 & 9.36 & 0.674 & \multirow{2}{*}{32.500} & \multirow[b]{2}{*}{.065} \\
\hline & Brasil & 11 & 9.91 & 0.302 & & & 11 & 9.91 & 0.302 & & \\
\hline \multirow{2}{*}{59} & España & 11 & 9.18 & 0.874 & \multirow{2}{*}{35.500} & \multirow{2}{*}{.101} & 11 & 8.73 & 0.905 & \multirow{2}{*}{$22.000 *$} & \multirow{2}{*}{.010} \\
\hline & Brasil & 11 & 9.64 & 0.924 & & & 11 & 9.73 & 0.647 & & \\
\hline \multirow{2}{*}{60} & España & 11 & 8.45 & 1.695 & \multirow{2}{*}{36.500} & \multirow{2}{*}{.116} & 11 & 7.91 & 2.119 & \multirow{2}{*}{34.000} & \multirow{2}{*}{.088} \\
\hline & Brasil & 11 & 9.18 & 1.401 & & & 11 & 8.91 & 2.023 & & \\
\hline \multirow{2}{*}{61} & España & 11 & 9.09 & 0.944 & \multirow{2}{*}{$26.500 *$} & \multirow{2}{*}{.013} & 11 & 9.09 & 0.701 & \multirow{2}{*}{$21.000^{* *}$} & \multirow{2}{*}{.008} \\
\hline & Brasil & 11 & 9.91 & 0.302 & & & 11 & 9.91 & 0.302 & & \\
\hline
\end{tabular}

\section{DISCUSIÓN Y CONCLUSIONES}

Los resultados obtenidos manifiestan la existencia de diferencias en la autopercepción valorativa por parte del profesorado en el ámbito de la asunción y desarrollo de las Habilidades Sociales. El profesorado universitario de España manifiesta un grado de autopercepción más bajo que el profesorado de Brasil, si bien se trata de diferencias muy poco significativas.

En este sentido, podríamos decir, que cada profesor, sea de España o Brasil, tiene su estilo propio de gestionar y desarrollar los procesos de convivencia social en sus aulas. Aunque el profesorado de España en esta investigación, presenta una menor 
puntuación en las variables principales de los estilos de enseñanza que el profesorado de Brasil.

Este resultado puede ser explicado por las diferencias existentes entre los dos países, como: culturales, económicas, geográficas y jurídicas, entre otras, como también, por las diferencias en los tipos de centro de trabajo del profesorado, ya que en España el profesorado universitario participante ha sido en su totalidad de centros de titularidad pública, mientras que en Brasil únicamente el $15 \%$.

Teniendo en cuenta todos estos resultados, podríamos afirmar que para el desarrollo global del constructo teórico-conceptual de "estilos de enseñanza universitaria" se precisaría la inclusión e integración de variables psicosociales, contextuales y de proceso, partiendo de la consideración de que el estilo de enseñar "es el modo habitual de acercase a los alumnos utilizando variados métodos de enseñanza. Se desprende de ello la importancia de la forma como el docente establece relaciones de “enseñanza”, con sus alumnos” (Herrera Márquez, 2005, p. 161).

El diseño de una propuesta conceptual de Estilo de Enseñanza, a partir de la percepción del profesorado de Enseñanza Superior de dos países distintos, como España y Brasil, en los aspectos, entre otros, económicos, culturales y geográficos ha permitido un análisis descriptivo transcultural del papel de la enseñanza universitaria en relación a la sociedad (Díaz y Martins, 2008), mostrando un perfil competencial actualizado del docente universitario y su relación con la sociedad. La definición de su papel nos ofrece una orientación esencial para el tipo de metodología de enseñanza a ser adoptada e instaurada para convertir a las instituciones de Educación Superior en centros de desarrollo profesional y social más ajustadas y adaptadas a las exigencias y demandas de su entorno.

Así pues, el profesorado que se ha preparado bien para impartir conocimientos de sus materias académicas, se ve abocado a responsabilizarse de un grupo de alumnos, que le plantea muchas veces retos ajenos a las lecciones que imparte. Poco a poco, el profesor descubre que no es suficiente su buena preparación académica, porque otros problemas de comportamiento, actitudes y relaciones, desbordan sus planes. Problemas sociales, afectivos y emocionales se entrecruzan en el devenir de la vida de los centros y de las aulas, y no siempre se está preparado suficientemente para hacerlos frente y solucionarlos (Carbonero, Román, Martín-Antón y Reoyo, 2009).

Con frecuencia, ni siquiera es posible detectarlos con claridad, por eso se hace necesario adoptar una perspectiva amplia, que admita la participación de un conjunto grande de habilidades y destrezas para abordar los nuevos problemas que presenta la actividad docente y la dinámica convivencial en las aulas y en los centros educativos (Martín-Antón, Carbonero y Román, 2012). Cada vez se considera más importante prestar atención al factor ambiental, en el que tiene lugar la enseñanza y el aprendizaje, como ámbito específico en que las actividades adquieren su sentido humano. 


\section{REFERENCIAS}

Bainbridge, L. (1999). Verbal reports as evidence of the process operator's knowledge. International Journal of Human-Computer Studies, 51(2), 213-238.

Braskamp, L.A.; Caulley, D. y Costin, F. (1979). Student ratings and instructor self-ratings and their relationship to student achievement. American Educational Research Journal, 16, 295-306.

Carbonero, M.A.; Román, J.M.; Martín-Antón, L.J. y Reoyo, N. (2009). Efecto del programa de habilidades docentes motivadoras en el profesorado de Secundaria. Revista de Psicodidáctica. 14(2), 229-244.

Díaz, J.B. y Martins, A.P. (2008). Estratégias de ensino aprendizagem. (29 ed.). Petrópolis, RJ, Brasil: Vozes.

Gana, K. y Brechenmacher, N. (2001). Structure letente et validité de la version française du SelfMonitoring Scale: échelle de monitorage de soi//Latent, structure and validity of the French version of the self-monitoring scale. L'année psychologique. 101(3), 393-420.

Gargallo, B., Suárez, J., Garfella, P.R., Fernández, A. (2012). El cuestionario CEMEDEPU. Un instrumento para la evaluación de la metodología docente y evaluativa de los profesores universitarios / The TAMUFQ Questionnaire (Teaching and Assessment Methodology of University Faculty Questionnaire). An Instrument to Asses the Teaching Methodology of University Faculty. ESE. Estudios sobre educación. 21, 49-71. Recuperado de http://hdl.handle.net/10171/22511 (el 23/10/2012).

Guillen, F. (1992). Autopercepción y evaluación del profesor según diferentes niveles de enseñanza. Revista Interuniversitaria de Formación del Profesorado, 14, 137-148.

Herrera, M.M. (2005). Estilo de Enseñar y aprender en la formación inicial y permanente del profesorado Venezolano. (Tesis doctoral inédita). Universidad de Valladolid, Valladolid.

Hervás, R.M. (2005). Estilos de enseñanza y de aprendizaje en escenarios educativos. Granada: Grupo editorial universitario.

Kerlinger, F.N. (1985). Enfoque conceptual de la investigación del comportamiento. Nueva editorial Interamericana. Mexico.

Maintier, C. y Alaphilippe, D. (2006). Validation d'un questionnaire d'auto-évaluation de soi destiné aux enfants. L'année psycologique, 106(4), 513-542.

Marshall, W.; Overall, J. y Kesler, S. (1979). Validity of student evaluation of Instructional effectiveness: A comparison of Faculty Self-evaluations and Evaluations by their students. Journal of Educational Psychology, 71, 149-160.

Martín-Antón, L.J.; Carbonero, M.A. y Román, J.M. (2012). Efecto modulador de variables socioemocionales en el entrenamiento de estrategias de elaboración en Educación Secundaria Obligatoria (ESO): paráfrasis y aplicaciones. Psicothema, 24(1), 35-41.

McGuigan, F.J. (1996). Psicología experimental, métodos de investigación. México: Hispanoamericana S.A. Cap. 12.

Normand, R. y Derouet, J.L. (2011). Évaluation, développement professionnel et organisation scolaire. Revue française de pédagogie. 174. 5-20.

Ovejero, A. y otros (1982). Detección de actitudes hacia el propio perfeccionamiento del profesorado. Documentación Enseñanzas Integradas, 1, 31-37.

Pesquero, E., Sánchez, E. y otros (2008). Las competencias profesionales de los Maestros de Primaria. Revista Española de Pedagogía, 241, 447-466.

Romuald, N. y Derouet, J.L. (2011). Évaluation, development professionnel et organisation scolaire. Revue Française de pédagogie. 174 (janvier-février-mars).

Torrano, F. y González, M.C. (2004). Self-regulated learning: current and future directions. Electronic Journal of Research in Educational Psychology, 2(1), 1-34. 
Winne, P.H. y Perry, N.E. (2000). Measuring self-regulated learning. En M. Boekaerts, P.R., Pintrich y M. Zeidner (eds.), Handbook of self-regulation (pp. 531-566). San Diego, California: Academic Press.

Yu, S. y Ding, R.J. (2009). Multi-level Fuzzy Assessment Application in Teaching Evaluation. ICAIE 2009: Proccdedings of the 2009 International Conference on Artificial Intelligence and Education, (1-2), 94-98. Huazhong NormalUniv. China Assoc Educ. Technol.

Recibido: 18 de febrero de 2013

Recepción Modificaciones: 8 de marzo de 2013

Aceptado: 19 de marzo de 2013 